\title{
The chiral phase transition for QCD with sextet quarks
}

\section{K. Sinclair ${ }^{* \dagger}$}

HEP Division, Argonne National Laboratory, 9700 South Cass Avenue, Argonne, IL, 60439, USA

E-mail: dks@hep.anl.gov

\section{J. B. Kogut}

Department of Energy, Division of High Energy Physics, Washington, DC 20585, USA

and

Department of Physics - TQHN, University of Maryland, 82 Regents Drive, College Park, MD

20742, USA

E-mail: jbkoguteumd.edu

QCD with 2 massless colour-sextet quarks is studied as a model of Walking Technicolor. We simulate lattice QCD with 2 light color-sextet staggered quarks at finite temperature, and use the dependence of the coupling at the chiral transition on the temporal extent, $N_{t}$, of the lattice in lattice units to study the running of the bare lattice coupling with lattice spacing. Our goal is to determine whether this theory is QCD-like and 'walks', or if it is conformal. If it is QCDlike, the coupling at the chiral transition should tend to zero as $N_{t} \rightarrow \infty$ in a manner controlled by asymptotic freedom, i.e. by the perturbative $\beta$-function. On the other hand, if this theory is conformal, this coupling will approach a non-zero limit in the $N_{t} \rightarrow \infty$ limit. We are extending our simulations on an $N_{t}=8$ lattice to determine the position of the chiral transition with greater accuracy, and are performing simulations on an $N_{t}=12$ lattice.

XXIX International Symposium on Lattice Field Theory

July 10-16 2011

Squaw Valley, Lake Tahoe, California

\footnotetext{
* Speaker.

$\dagger$ This research was supported in part by US Department of Energy contract DE-AC02-06CH11357.

${ }^{\ddagger}$ Supported in part by a National Science Foundation grant NSF PHY03-04252.
} 


\section{Introduction}

We are interested in extensions of the Standard Model with strongly-interacting Higgs sectors. The most promising theories of this type are Technicolor models [1, 2]. Technicolor theories are QCD-like theories where the techni-pions play the rôle of the Higgs field, giving masses to the $W$ and $Z$. Walking Technicolor theories, where the fermion content is such that the coupling constant evolves very slowly - 'walks' - and their extensions can avoid phenomenological problems, which otherwise afflict Technicolor models [3, 月, 5, 6].

QCD with $1 \frac{28}{125} \leq N_{f}<3 \frac{3}{10}$ flavours of massless colour-sextet quarks is expected to be either a Walking or a Conformal field theory. (First term in the $\beta$ function is negative, second positive.) For $N_{f}=3$ conformal behaviour is expected. $N_{f}=2$ could, a priori, exhibit either behaviour. We use unimproved lattice QCD with staggered quarks to simulate these theories. The RHMC simulation algorithm [7] is used to allow us to simulate at values of $N_{f}$ which are not multiples of 4.

We simulate $N_{f}=2$ thermodynamics in an attempt to distinguish whether it is walking or conformal. Simulations on $N_{t}=4,6,8$ lattices show widely separated deconfinement and chiral symmetry restoration transitions, which move to weaker couplings as $N_{t}$ is increased [8, 9]. For the chiral transition, the separation between the $N_{t}=8$ and $N_{t}=6$ transitions is considerably less than that between the $N_{t}=6$ and $N_{t}=4$ transitions. 2-loop perturbation theory suggests that between $N_{t}=8$ and $N_{t}=6$ the shift is consistent with asymptotic freedom, while between $N_{t}=6$ and $N_{t}=4$ the theory is strongly-coupled and the shift is due to quenched evolution of the coupling. If this is correct, the deconfinement transitions occur in the strong-coupling (quenched) regime for any $N_{t}$ we could reasonably study. We are thus limiting our studies to the evolution of the couplings at the chiral transition as $N_{t}$ is increased. Other groups are studying QCD with 2 colour-sextet quarks using different lattice actions [10, 11, 12, 13, 14, 15, 16, 17,. Most of these studies are of the zero-temperature behaviour of this theory. A consensus as to whether this theory is QCD-like or conformal has yet to be reached.

We are now extending our simulations to $N_{t}=12$ lattices and plan simulations on $N_{t}=18$ lattices, to check if this theory is indeed QCD-like, or whether we are observing the approach to a bulk chiral transition, expected for a conformal theory. (Our simulations are limited to the neighbourhood of the chiral transition - weak coupling.) Since, however, 2-loop perturbation theory predicts that $\beta_{\chi}\left(N_{f}=12\right)-\beta_{\chi}\left(N_{f}=8\right) \approx 0.12$, we first need to increase the number of $\beta$ values and statistics in the neighbourhood of the chiral transition, for $N_{t}=8$. We are also adding simulations at a smaller quark mass, $m=0.0025$. These additional $N_{t}=8$ runs also provide evidence that the $N_{f}=2$ chiral transition is second order.

\section{Simulations at $N_{t}=8$}

We are extending our simulations on $16^{3} \times 8$ lattices in the neighbourhood of the chiral transition. Our quark masses are $m=0.0025, m=0.005, m=0.01, m=0.02$, which will allow us to access the chiral limit. Whereas our earlier simulations used $\beta$ values separated by 0.1 , we have decreased this to 0.02 close to the transition. Near this transition we use 50,000-trajectory runs at each $(\beta, m)$ for $m=0.0025,20,000$-trajectory runs for $m=0.005$ and $m=0.01$ and 10,000- 
trajectory runs at $m=0.02$. We are currently increasing or plan to increase our run lengths at each of these masses.

The chiral condensates for each mass decrease as $\beta$ increases. More importantly, as $\beta$ increases, the mass dependence of these condensates becomes more pronounced. The decrease in the chiral condensate with decreasing mass is such that it does appear that it will vanish in the chiral limit for $\beta$ sufficiently large. However, the $\beta$ dependence of $\langle\bar{\psi} \psi\rangle$ is sufficiently smooth at all the masses of our simulations, that we would need a precise analytical form to perform a believable chiral $m \rightarrow 0$ extrapolation to determine where it vanishes. This we do not have. Hence we examine the (disconnected) chiral susceptibilities

$$
\chi_{\bar{\psi} \psi}=\frac{V}{T}\left[\left\langle(\bar{\psi} \psi)^{2}\right\rangle-(\langle\bar{\psi} \psi\rangle)^{2}\right]
$$

where $V$ is the spatial volume of the lattice and $T$ is the temperature. $\bar{\psi} \psi$ is a lattice averaged quantity. Because we only have stochastic estimators for $\bar{\psi} \psi$ (5 per trajectory), we obtain unbiased estimators of $(\bar{\psi} \psi)^{2}$ as the products of 2 different estimators of $\bar{\psi} \psi$ for the same gauge configuration.

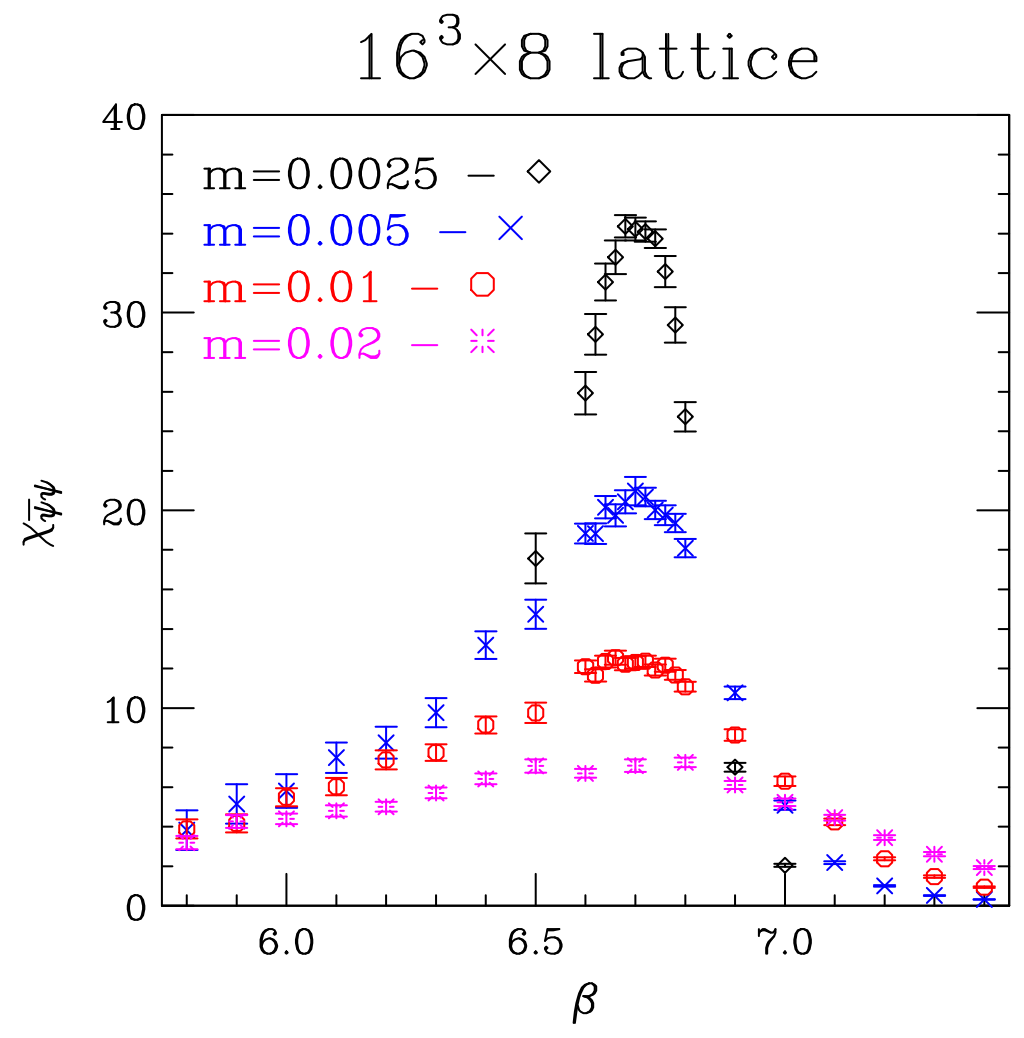

Figure 1: Chiral susceptibilities on a $16^{3} \times 8$ lattice.

The chiral susceptibility diverges at the chiral phase transition for zero quark mass. At small but finite mass, it shows a clear peak which becomes sharper as $m$ decreases. Extrapolating the position of said peak to $m=0$ yields $\beta_{\chi}$, the $\beta$ value of the chiral phase transition. Figure 11 shows the chiral susceptibilities from our runs on $16^{3} \times 8$ lattices. What is clear from this plot is that the 
position of the peak in the chiral susceptibility has very little dependence on the quark mass $m$. It is this fact that allows us to extrapolate its position to $m=0$. Our best estimate from the 'data' presented here is $\beta_{\chi}=6.70(2)$.

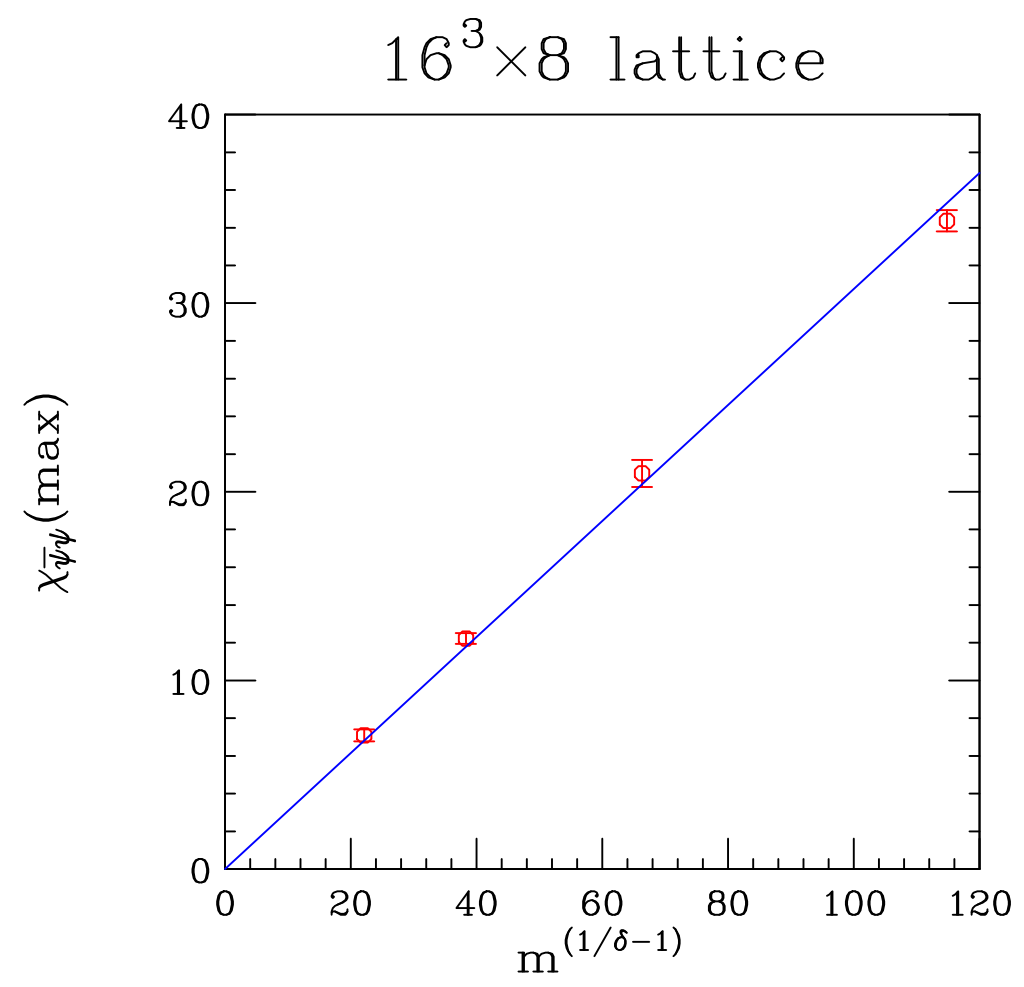

Figure 2: Peaks of the chiral susceptibilities for each mass as functions of the scaling variable $m^{1 / \delta-1}$.

Assuming that the chiral transition is a second order transition in the equivalence class of the 3-dimensional $O(2)$ or $O(4)$ spin model, as expected for a finite temperature transition, we fit the peaks of the susceptibilities to the scaling form

$$
\chi_{\bar{\psi} \psi}(\max )=C m^{1 / \delta-1}
$$

with $\delta=4.8$. Figure 2 shows that the fit is quite reasonable $-\chi^{2} / D O F \approx 2.2$. This contrasts to the fit to the form for a first-order transition $(\delta=\infty)$ which is very poor $-\chi^{2} / D O F \approx 65$.

\section{Simulations at $N_{t}=12$}

Our $N_{t}=12$ simulations are being run on a $24^{3} \times 12$ lattice for quark masses $m=0.01, m=$ 0.005 and $m=0.0025$ and a range of $\beta$ values which includes the chiral transition. We perform runs of 10,000 length- 1 trajectories away from this transition. Close to the transition $(6.6 \leq \beta \leq 6.9)$ the $\beta$ values are spaced by 0.02 , and we plan runs of 50,000 trajectories for each $(\beta, m)$. The results we are reporting have 10,000-25,000 trajectories at each $(\beta, m)$.

Figure 3 shows preliminary measurements of the chiral susceptibilities obtained in these simulations. As one can see, the susceptibility rises rapidly as $\beta$ is decreased from $\beta=7.2$ down to $\beta$ just above 6.8 , for each of the 3 mass values. Below this each of the graphs flattens out. This is 


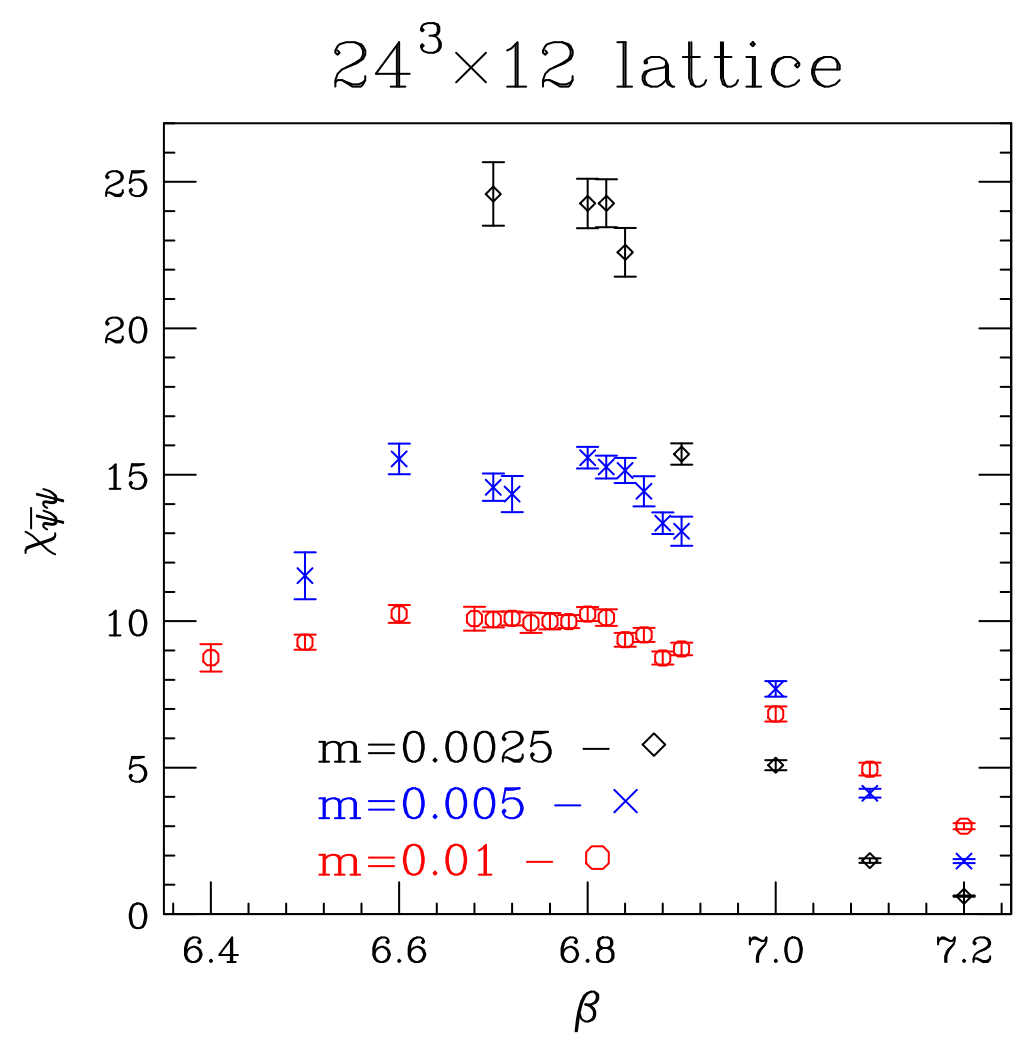

Figure 3: Chiral susceptibilities on a $24^{3} \times 12$ lattice.

obvious for $m=0.01,0.005$, and there is some preliminary evidence for this at $m=0.0025$. From this preliminary 'data' we cannot estimate the position of the chiral transition. For this we will need higher statistics and possibly a lower mass value.

Since we cannot as yet estimate the position $\beta_{\chi}$ of the chiral transition from the chiral susceptibilities, we perform a direct comparison between the chiral condensates on the $16^{3} \times 8$ and $24^{3} \times 12$ lattices for the same mass. Ideally we should select the smallest mass $(m=0.0025)$. However, we do not yet have enough $\beta$ values at $m=0.0025$ to make a good comparison. Hence we compare the chiral condensates at $m=0.005$ on the two lattices. Figure 4 shows this comparison. The $24^{3} \times 12$ condensates are displaced to larger $\beta$ with respect to the $16^{3} \times 8$ condensates. However, the displacement appears to be less than the $\approx 0.12$ predicted from the 2-loop Callan-Symanzik beta-function.

\section{Discussion and conclusions}

We are simulating lattice QCD with 2 flavours of light staggered quarks at finite temperatures on $16^{3} \times 8$ and $24^{3} \times 12$ lattices for $\beta$ close to the chiral transition. Our goal is to determine whether the chiral transition for massless fermions approaches zero coupling (infinite $\beta$ ) as $N_{t} \rightarrow \infty$ in a manner predicted by the perturbative $\beta$-function, implying a QCD-like (walking) theory, or if it approaches a finite non-zero value indicating a conformal field theory. For $N_{t}=8$ we are able to determine the position of the chiral transition $\beta_{\chi}$ with some accuracy $\left(\beta_{\chi}=6.70(2)\right)$ from the peaks 


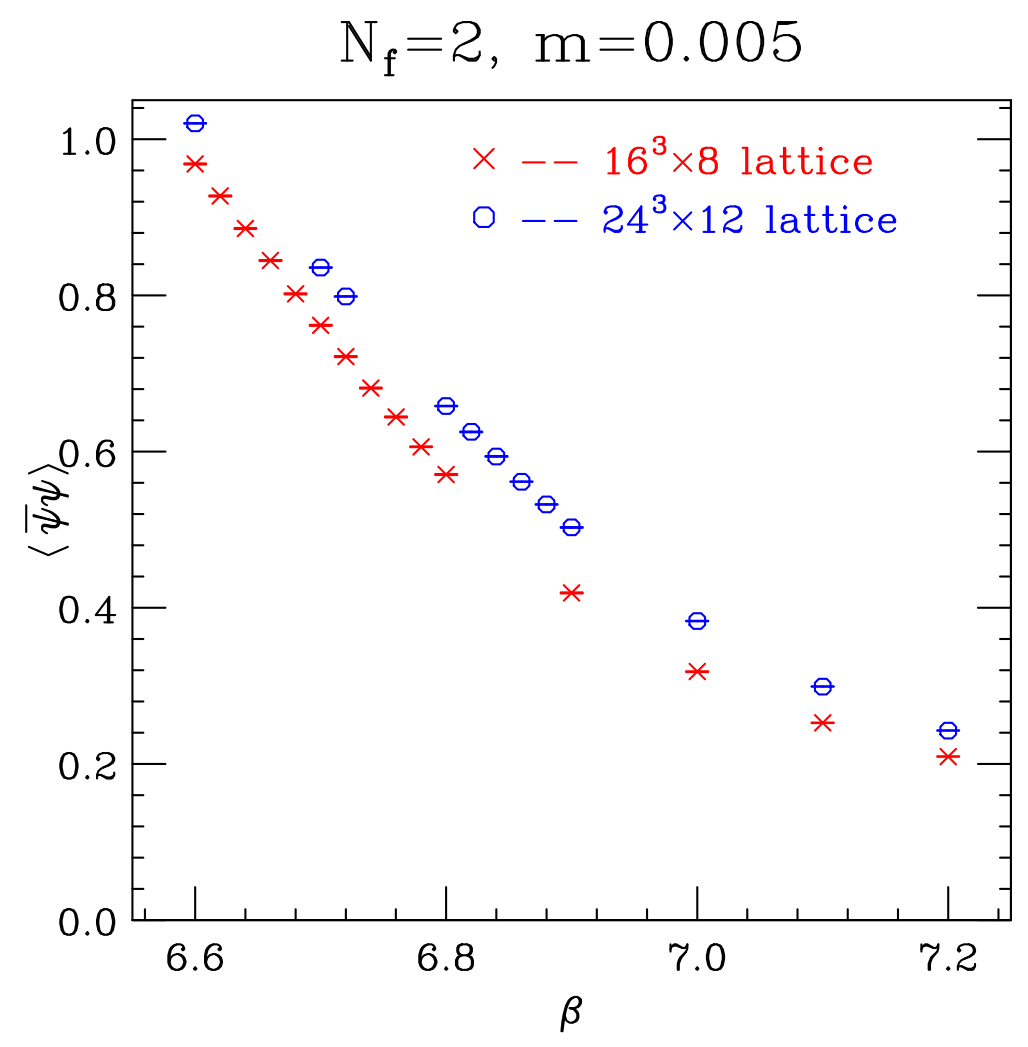

Figure 4: The $m=0.005$ chiral condensates on $16^{3} \times 8$ and $24^{3} \times 12$ lattices as functions of $\beta$.

in the chiral susceptibilities. The scaling of these peaks with mass is consistent with a second-order phase transition in the $O(2) / O(4)$ universality class, expected of a finite-temperature transition, and inconsistent with the first-order transition expected for a bulk transition. For $N_{t}=12$ our 'data' is still too preliminary to determine $\beta_{\chi}$ from the chiral susceptibilities. However, direct comparison of the chiral condensates suggests that $\beta_{\chi}\left(N_{t}=12\right)>\beta_{\chi}\left(N_{t}=8\right)$ as predicted by asymptotic freedom for a finite temperature transition.

For $N_{t}=8$, we have also performed simulations on a $24^{3} \times 8$ lattice at a few strategically chosen values of $(\beta, m)$. Comparison of the chiral condensates and chiral susceptibilities between the 2 spatial volumes indicates that the finite volume effects are small.

For $N_{t}=12$, we need more statistics at more values of $\beta$ for each mass, to determine the peaks in the chiral susceptibilities. If the susceptibilities remain too flat to be able to determine their peaks, we may need to simulate at an even smaller mass $(m=0.00125)$ to determine $\beta_{\chi}$. We will also need to check that the finite volume effects are under control. We see no reason why results as precise as those we obtained on $N_{t}=8$ lattices cannot be obtained.

It is probable that simulations at even larger $N_{t} \mathrm{~s}$ will be needed to resolve the true nature of this theory. We are planning simulations at $N_{t}=18\left(36^{3} \times 18\right.$ lattices $)$. In addition we are planning some zero temperature simulations in the chirally-restored phase. 


\section{Acknowledgements}

These simulations were performed on the Cray XT6 - Hopper, the Cray XT4 - Franklin and the Linux Cluster - Carver/Magellan at NERSC, on the Cray XT5 - Kraken at NICS under a TRAC allocation and on the LCRC's Linux Cluster - Fusion at Argonne National Laboratory.

\section{References}

[1] S. Weinberg, Phys. Rev. D 19, 1277 (1979).

[2] L. Susskind, Phys. Rev. D 20, 2619 (1979).

[3] B. Holdom, Phys. Rev. D 24, 1441 (1981).

[4] K. Yamawaki, M. Bando and K. i. Matumoto, Phys. Rev. Lett. 56, 1335 (1986).

[5] T. Akiba and T. Yanagida, Phys. Lett. B 169, 432 (1986).

[6] T. W. Appelquist, D. Karabali and L. C. R. Wijewardhana, Phys. Rev. Lett. 57, 957 (1986).

[7] M. A. Clark and A. D. Kennedy, Phys. Rev. D 75, 011502 (2007) [arXiv:hep-lat/0610047].

[8] J. B. Kogut, D. K. Sinclair, Phys. Rev. D81, 114507 (2010). [arXiv:1002.2988 [hep-lat]].

[9] J. B. Kogut, D. K. Sinclair, [arXiv:1105.3749 [hep-lat]].

[10] Y. Shamir, B. Svetitsky and T. DeGrand, Phys. Rev. D 78, 031502 (2008) [arXiv:0803.1707 [hep-lat]].

[11] T. DeGrand, Y. Shamir and B. Svetitsky, Phys. Rev. D 79, 034501 (2009) [arXiv:0812.1427 [hep-lat]].

[12] T. DeGrand, Phys. Rev. D 80, 114507 (2009) [arXiv:0910.3072 [hep-lat]].

[13] T. DeGrand, Y. Shamir and B. Svetitsky, Phys. Rev. D 82, 054503 (2010) [arXiv:1006.0707 [hep-lat]].

[14] T. DeGrand, Talk presented at Lattice2011, Squaw Valley, California (2011).

[15] Z. Fodor, K. Holland, J. Kuti, D. Nogradi and C. Schroeder, PoS LATTICE2008, 058 (2008) arXiv:0809.4888 [hep-lat].

[16] Z. Fodor, K. Holland, J. Kuti, D. Nogradi and C. Schroeder, arXiv:1103.5998 [hep-lat].

[17] J. Kuti, Talk presented at Lattice2011, Squaw Valley, California (2011). 